\title{
Perceptual grouping in good and poor readers
}

\author{
MARY C. WILLIAMS and NANCY B. BOLOGNA \\ University of New Orleans, New Orleans, Louisiana
}

\begin{abstract}
A selective attention task was used to measure perceptual grouping processes in 9- to 10-yearold children who were classified as good, average, and poor readers. Results showed that there is a negative correlation between perceptual grouping effects and reading ability: Grouping ef fects are stronger for poor readers. This relationship is maintained whether or not an optimal strategy for performing the selective attention task is provided.
\end{abstract}

Reading involves a visual processing task that requires the analysis of features of visual patterns. Studies in the area of reading have investigated visual processing deficits that may be involved in reading disability. Several studies have indicated that normal and disabled readers do not show differences in basic visual processes (Vellutino, Pruzek, Steger, \& Meshoulam, 1973; Vellutino, Steger, DeSetto, \& Phillips, 1975; Vellutino, Steger, Kaman, \& DeSetto, 1975; see Vellutino, 1977, for a review). Other studies have found evidence for a specific visuoperceptual disorder in certain types of reading disability (CliftonEverest, 1976; Critchley, 1964; Natchez \& Roswell, 1964). Studies such as these have examined processing at several different levels of the visual system, which may, in part, account for the discrepancy in findings.

Several researchers have looked specifically at differences between normal and disabled readers at early stages of visual processing. Differences have been reported in visual-information-store duration (Lovegrove \& Brown, 1978; Stanley, 1975; Stanley \& Hall, 1973b), in rate of transfer of information from visual information store to short-term memory (Lovegrove \& Brown, 1978; Stanley \& Hall, 1973b), and in the characteristics of visual short-term memory itself (Stanley \& Hall, 1973a). These results indicate that some disabled readers process information more slowly and have a more limited capacity than normal readers. Recent work has shown that disabled readers show deficits in a transient subsystem that is a fast, early processing system that mediates global-form processing and movement (see Lovegrove, Martin, \& Slaghuis, 1985, for a review).

The work reported here involves a perceptual operation that occurs very early in the visual processing sequence-perceptual grouping. Perceptual grouping refers to the linking or segmenting of elements into figures and regions. The classical studies of perceptual grouping were conducted by the Gestalt psychologists, who formulated a number of laws of perceptual organization, includ-

\footnotetext{
The authors wish to thank James G. May for thoughtful critical advice throughout. Reprint requests should be sent to the first author. Department of Psychology, University of New Orleans. New Orleans. LA 70148 .
}

ing the laws of similarity, proximity, continuity, and Prägnanz. These laws specified conditions for perceptual grouping. For example, it was demonstrated that elements that are similar to one another, or close in space to one another, will group into the same perceptual unit. Although useful for demonstrating the existence of such perceptual effects, the Gestalt method of study (the phenomenological method) was entirely subjective; it provided no mechanism for objectively measuring the dynamics or underlying processes of perceptual grouping operations.

Recently, Pomerantz and his coworkers (Pomerantz \& Garner, 1973; Pomerantz \& Schwaitzberg, 1975; see Pomerantz, 1977, for a review) operationalized the study of perceptual grouping. They employed a speeded classification task in which normal adult subjects were presented with two-element stimuli. The subjects' task was to classify the stimuli on the basis of variation of both elements or of only one element with the second held constant. The logic behind this task is that if the two elements belong to the same perceptual unit, it should be difficult to selectively attend to one relevant element and ignore (filter) the other. If, on the other hand, the two elements belong to separate perceptual groups, it should be easy to selectively attend to one element and ignore the other. Thus, perceptual grouping can be objectively evidenced by the failure of selective attention.

Given that perceptual grouping can be objectively studied, it would seem to be of particular interest to apply it to the study of reading. The perceptual organization of separate elements into unitary wholes must necessarily be involved in the initial stages of the reading process. As mentioned above, good and poor readers have shown differences in early visual operations, especially those associated with the transient subsystem, and perceptual grouping has been associated with transient activity (Williams \& Weisstein, 1980). Perhaps perceptual grouping operations are a heretofore unidentified problem area for disabled readers. To investigate this possibility, we applied the procedure developed by Pomerantz and his coworkers to the study of children with good, average, and poor reading ability. 
EXPERIMENT 1A: Selective Attention Measures of Perceptual Grouping

In Experiment $1 \mathrm{~A}$ we measured selective attention operations with stimuli that have previously been shown to produce perceptual grouping effects. As mentioned above, when two elements belong to the same perceptual unit, it should be difficult to selectively attend to just one element. The purpose of the present experiment was to determine whether good and poor readers show differences in their ability for selective attention to elements in the same perceptual unit.

\section{Method}

\section{Subjects}

Twenty-three fourth-grade children, 9 boys and 14 girls aged 9 to 10 years, participated as subjects. All had average or aboveaverage IQs and normal or corrected-to-normal vision; all were initially screened with the Spache (1981) Diagnostic Reading Scales (DRS) to establish reading ability levels. The DRS independent level, a measure of silent reading ability, was used to place subjects. ${ }^{1}$ Of the 23 subjects, 8 placed at the fourth-grade level, 9 placed at least one grade level below, and 6 placed at least one grade level above.

\section{Stimuli}

For purposes of comparability, we used a stimulus set similar to that used by Pomerantz and Garner (1973) and Pomerantz and Schwaitzberg (1975). The four stimuli used in the present experiment are shown in Figure 1. The bracket stimuli were drawn with black India ink on white paper, photocopied, and placed on $7.6 \times 6.4 \mathrm{~cm}$ white cards. One bracket pair was positioned in the center of each card. Each bracket was $8 \mathrm{~mm}$ tall and $4 \mathrm{~mm}$ wide, and the spacing between the brackets was $4 \mathrm{~mm}$. At a typical viewing distance of $30 \mathrm{~cm}$, the stimuli subtended a viewing angle of approximately $1.5^{\circ}$.

\section{Conditions}

Stimuli were arranged into four decks of 32 cards each. Each deck represented a different experimental condition. The task used was speeded card sorting.

\section{Control Condition}

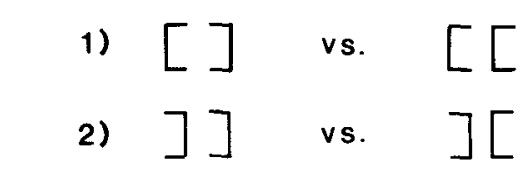

Orthogonal Condition



Figure 1. The two experimental conditions in Experiment 1A. Control condition (A): left (irrelevant) element does not vary; sort can be accomplished on the basis of right (relevant) element alone. Orthogonal condition (B): left (irrelevant) element varies orthogonally to the right (relevant) element; sort can be accomplished via selective attention to the right element.
Control conditions. There were two control conditions in which only the relevant element varied whereas the irrelevant element remained constant. Subjects sorted cards on the basis of the relevant element. Each of the two decks contained only two stimuli. One deck contained the stimuli [] and [[, 16 of each, and the other contained the stimuli ]] and ][, again 16 of each. The subjects' task was to sort the cards into two piles (see Figure 1A). In both cases the left element does not vary; it is irrelevant to the task. The card sort can be accomplished on the basis of the right (relevant) element alone.

Orthogonal condition. In the orthogonal condition both the right and left elements varied, but in an independent or orthogonal manner. The deck of 32 cards contained all four stimuli in Figure 1, eight of each. Subjects sorted the cards on the basis of the variation of the right (relevant) element; the left (irrelevant) element varied orthogonally. The stimuli [] and ]] were to be sorted into one pile, and the stimuli [[ and ][ were to be sorted into the other pile (see Figure 1B). This was, therefore, a selective attention task. If subjects were able to selectively attend to the relevant element, then the variation of the irrelevant element should not interfere with sorting performance. Thus, if selective attention was possible, the sorting times in the control and orthogonal conditions should be similar. If selective attention was difficult or not possible, there should be interference from the irrelevant element in the orthogonal condition and sorting times should be longer. As mentioned above, the success or failure of selective attention is an index of whether and to what degree perceptual grouping occurs.

Color condition. To preclude the possibility that good and poor readers would show differences in visual-motor performance in general, we included an additional condition in which subjects sorted color-coded cards. A deck of 32 cards was used, 16 with a blue dot in the middle and 16 with a purple dot in the middle. The subjects' task was to sort the cards into two piles. This condition provided an index of differences in overall performance in the cardsorting task, independent of perceptual grouping effects.

\section{Procedure}

The task in all conditions was speeded classification (sorting) of a deck of cards into two piles. In each condition, the subject was handed a deck of cards along with an instruction card indicating which stimuli were to be put into which response pile. Subjects were allowed to hold the deck of cards in whichever hand they preferred, ${ }^{2}$ and were instructed to sort as fast as possible without making any errors. Sorting time was recorded by the experimenter with a stopwatch. Classification errors were also recorded, but were not analyzed, as they were rare and unsystematic. Subjects were not instructed concerning sorting strategies: they were left to develop a strategy on their own. Each subject received one practice trial in each condition, then sorted through each of the decks once. The order of the conditions was counterbalanced across subjects.

\section{Results and Discussion}

\section{Color condition}

Mean sorting times in the color condition were 23.85 , 24.75 , and $22.48 \mathrm{sec}$ for subjects with low, average, and high reading ability levels, respectively. A one-way ANOVA with unequal ns showed that these sorting times did not significantly differ $[F(2,20)=0.77, p=0.48]$. Thus, there is no evidence for differential overall performance in the card-sorting task across subject populations.

\section{Overall performance}

The data for the control and orthogonal conditions, averaged across all subjects, are shown in Figure 2. The overall trends in sorting times agree with those reported 


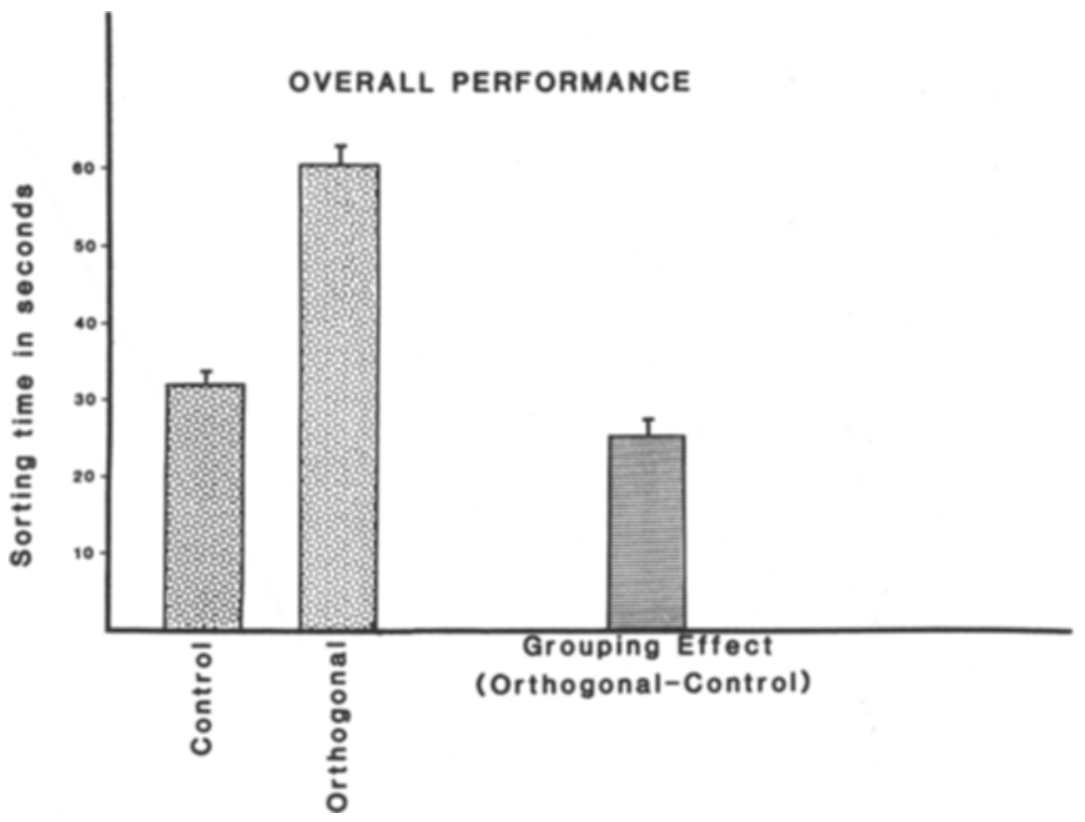

Figure 2. Sorting times (in seconds) for control and orthogonal conditions in Experiment 1A, averaged across all subjects. The grouping effect is the difference in sorting times between orthogonal and control conditions. Variance indicators represent 1 SEM.

by Pomerantz and Schwaitzberg (1975), with the sorting times for the control task being faster than those for the orthogonal task. A one-way ANOVA with unequal ns showed the main effect of experimental condition to be significant $[\mathrm{F}(2,44)=104.64, \mathrm{p}<.001]$.

Pairwise contrasts showed that the two control tasks did not significantly differ $[F(1,22)=3.49, \mathrm{p}>.05]$; data from these two tasks have been pooled in all subsequent analyses. Sorting times in the orthogonal condition were, however, significantly longer than in the control condition $[\mathrm{F}(1,22)=118.96, \mathrm{p}<.001]$.

The difference in sorting time between the orthogonal task and the control task constitutes a measure of perceptual grouping. The longer sorting times in the orthogonal condition indicate that it was harder to classify stimuli when the irrelevant element varied orthogonally to the relevant one than when only the relevant element varied. In other words, subjects could not selectively attend to the relevant element without some interference from the irrelevant element. The failure of selective attention indicates that the elements grouped into the same perceptual unit. The magnitude of the grouping effect across all subjects was $25.74 \mathrm{sec}$ (see Figure 2).

\section{Performance With Respect to Reading Ability}

The failure of selective attention in the overall performance scores indicates that the stimulus set we used produces strong perceptual grouping effects. The next question is whether the grouping effect is related to reading ability. Figure 3 shows the sorting time data for subjects with low, average, and high reading ability levels.
As can be seen, the grouping effect increases as reading ability decreases. In fact, there is a significant negative correlation between grouping effect and reading scores $(r=-.48, p<.05)$. This is not to imply that the grouping tendency is a determinant of reading ability, but rather that perceptual grouping is another early visual operation that differs in good and poor readers. It is possible that a third factor, such as processing characteristics of the transient subsystem, affects both grouping effects and reading performance.

The grouping effect, although consistently related to reading level, failed to show significance in a one-way ANOVA of grouping effect at low, average, and high reading levels $[F(2,20)=1.94, p>.05]$. Inspection of the data made it clear that the lack of significance was probably related to the large variability in the reading ability of average readers, while the good and poor readers formed more homogeneous groups. For good (aboveaverage) and poor (below-average) readers, the grouping effect did differ significantly $[\mathrm{F}(1,13)=4.25$, $\mathrm{p}<.05$ ], with the poor readers showing a stronger grouping effect.

\section{EXPERIMENT 1B: Selective Attention in the Absence of Perceptual Grouping}

In Experiment $1 \mathrm{~A}$ we found that poor readers show stronger perceptual grouping effects than good readers. In fact, the magnitude of perceptual grouping increases as reading ability decreases. The larger grouping effect 


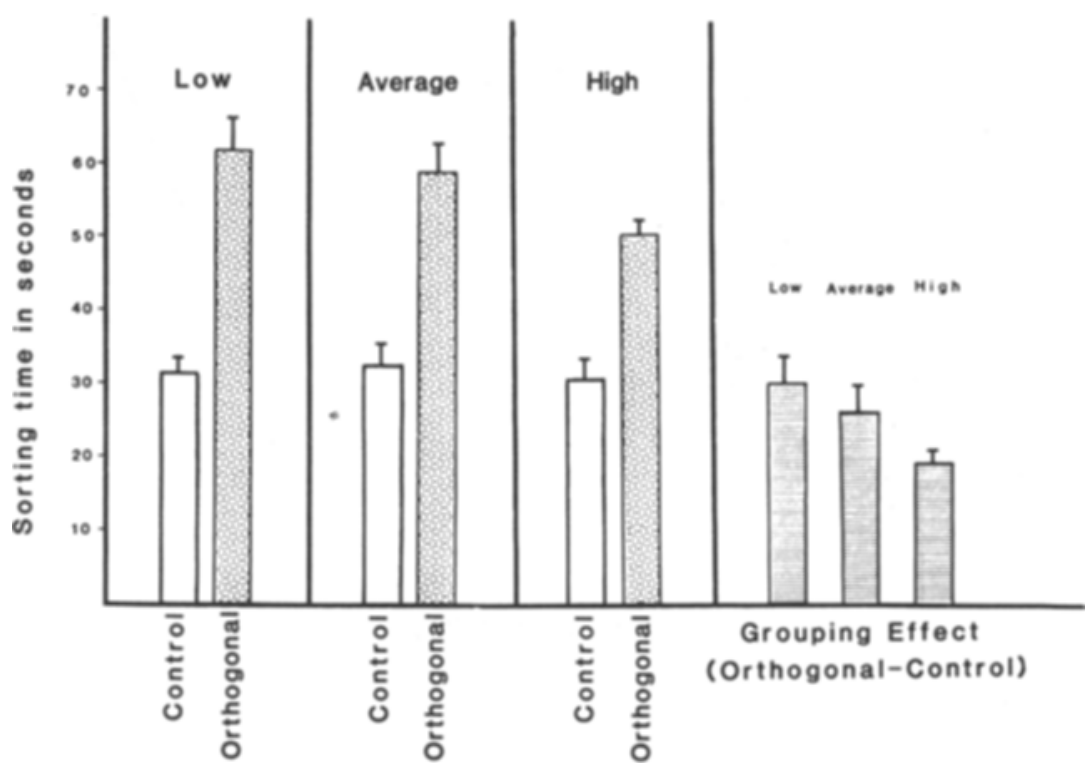

Figure 3. Sorting times for control and orthogonal conditions (in seconds) for subjects with low, average, and high silent reading ability levels. The grouping effect is the difference in sorting times between orthogonal and control conditions.

indicates that poor readers are less proficient at selective attention to elements within the same perceptual unit than are good readers. In Experiment 1B, we administered the same selective attention task, but used a stimulus set that did not produce perceptual grouping effects. As mentioned above, when two elements belong to separate perceptual groups, it should be easy to selectively attend to one element and ignore the other. The purpose of this experiment was to determine whether good and poor readers show differences in their ability to selectively attend to elements in separate perceptual units.

\section{Method}

Experiment 1B was identical to Experiment 1A, except that a different stimulus set was used (see Figure 4). These bracket stimuli comprised the same elements as those of Experiment $1 \mathrm{~A}$, but in a configuration that has previously been shown not to produce perceptual grouping effects (Pomerantz \& Garner, 1973). The bracket stimuli were arranged into three decks of cards corresponding to the three conditions used in Experiment 1A: two control conditions and one orthogonal condition (see Figure 4). The same subjects that participated in Experiment 1A participated in this experiment also.

\section{Results and Discussion}

The data for the control and orthogonal conditions, averaged across all subjects, are shown in Figure 5. Here, the overall trends in sorting times do not agree with those reported by Pomerantz and Garner (1973) for adult subjects. Although adults in that study showed no significant difference between sorting times in the control and orthogonal conditions, our subjects do show a significant difference $[F(1,22)=41.51, p<.001]$, with orthogonal sorting times being longer than control sorting times. This difference, however, did not significantly vary with respect to reading ability $[F(2,20)=2.20, p>.05]$, and does not significantly correlate with reading scores $(r=-.07, p>.05)$. These results suggest that the inconsistency with previous findings is related to developmental differences rather than to reading ability. Adults show no interference effect in the orthogonal condition; they are able to selectively attend to elements in separate perceptual groups. Children appear to be less proficient than adults at such operations, but this lack of proficiency is apparently not related to reading ability.

\section{CONTROL CONDTION}

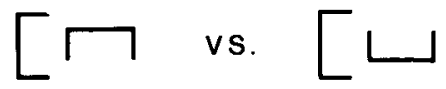

ORTHOGONAL CONDTION

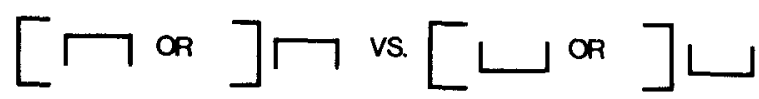

Figure 4. The two experimental conditions in Experiment 1B. The top row shows one of the control conditions: The left (irrelevant) element does not vary; sort can be accomplished on the basis of the right (relevant) element alone. The bottom row shows the orthogonal condition: The left (irrelevant) element varies orthogonally to the right (relevant) element; sort can be accomplished via selective attention to the right element. 


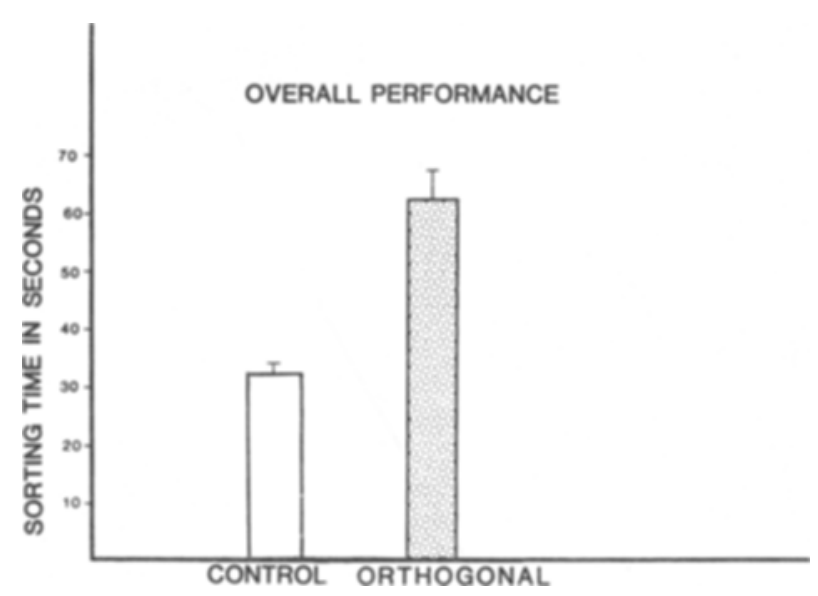

Figure 5. Sorting times (in sec) for control and orthogonal conditions in Experiment 1B, averaged across all subjects.

\section{EXPERIMENT 1C: Developmental Effects}

It is possible that the results of Experiment $1 \mathrm{~B}$ are due not to a true developmental effect, but rather to some artifact in our procedure that renders it incomparable with previous work with adults. To preclude this possibility, we ran adult subjects in the conditions of Experiments $1 \mathrm{~A}$ and $\mathrm{B}$ (control and orthogonal conditions with the stimulus sets shown in Figures 1 and 4). All procedures were identical to those described above. Four adults, aged 20 to 22 years, participated as subjects and were naive as to the purpose of the experiment.

\section{Results and Discussion}

The data obtained for the control and orthogonal conditions with the two stimulus sets are shown in Figure 6. A one-way ANOVA showed a significant main effect of experimental condition $[F(3,9)=15.0, p<.001]$. Pairwise contrasts showed that sorting times were significantly longer in the orthogonal condition than in the control condition $[F(1,3)=19.3, p=.025]$ with the stimulus set shown in Figure 1. The longer sorting times in the orthogonal condition indicate that subjects could not ignore the irrelevant element and sort on the basis of the right element. The failure of selective attention indicates that the elements grouped into the same perceptual unit.

Orthogonal and control sorting times did not, however, differ significantly with the stimulus set shown in Figure 4 $[F(1,3)=0.36, p>.05]$. More selective attention is possible when elements do not group into perceptual units. This is a replication of previous findings of Pomerantz and his coworkers (Pomerantz, 1977; Pomerantz \& Garner, 1973; Pomerantz \& Schwaitzberg, 1975). These results suggest that the difference between selective attention operations in children and in adults is due to a developmental effect and not to a procedural artifact. This finding is in agreement with the findings of others who have found age-related differences in perceptual group- ing and selective attention operations (Enns \& Girgus, 1985; Pick \& Frankel, 1973; Strutt, Anderson, \& Well, 1975; Vurpillot, 1976).

\section{EXPERIMENT 2: The Effect of Sorting Strategy}

In Experiment 1 we found two separate, although possibly related, effects. First, the grouping effect produced by the stimulus set in Figure 1 was related to reading ability. Second, there were performance differences between children and adults in selective attention operations with stimuli that did not group into perceptual units. This second effect, not related to reading ability, may be due to processing strategy differences. In the sorting tasks of Experiment 1 , subjects were not given the optimal strategy of sorting on the basis of the relevant element and ignoring the irrelevant element; they were left to develop a strategy on their own. Adults seemed to easily pick up the optimal strategy with stimuli that did not group. With stimuli that did group, however, the irrelevant element could not be ignored; it was in the same perceptual unit with the relevant one.

It is possible that the performance differences that we found between children and adults in the no-grouping condition were due to sorting strategy differences. Children may not spontaneously develop optimal processing strategies for selective attention, as adults seem to do. Furthermore, it is possible that those children in Experiment $1 \mathrm{~A}$ designated as good readers may have been more proficient than the poor readers in developing a strategy for selective attention. If, in fact, this was true, the results that indicated a difference in the perceptual grouping effect would be contaminated by differential ability to develop a sorting strategy. To investigate this possibility, we administered the same basic tasks of Experiment 1 to a new group of subjects, this time manipulating sorting strategies. The aim of Experiment 2 was (1) to identify

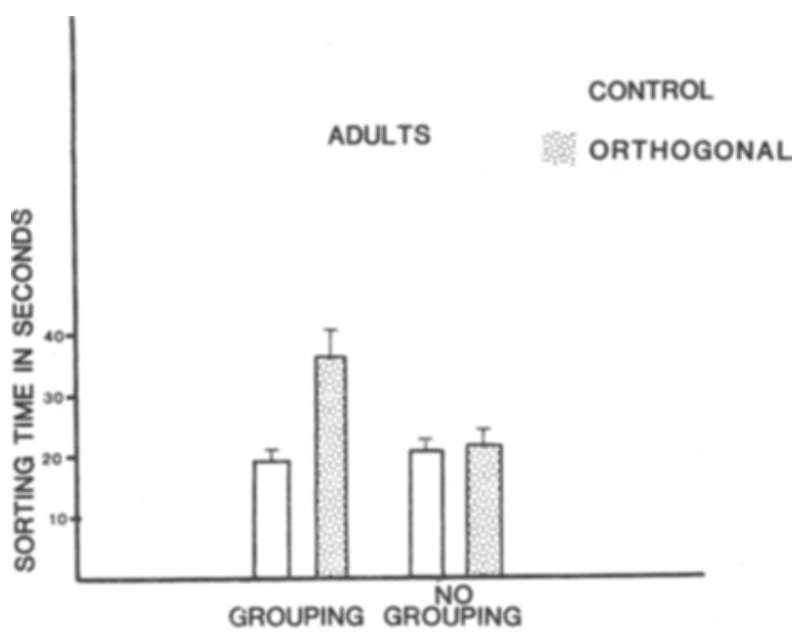

Figure 6. Sorting times (in sec) obtained with adult subjects in Experiment $1 \mathrm{C}$ for control and orthogonal conditions with the stimulus set that grouped (left) and with the one that did not (right). 
sorting strategy differences between children and adults, and (2) to evaluate separately the effects of strategy and selective attention operations with respect to reading ability.

\section{Method}

In Experiment 2, we administered the control and orthogonal sorting tasks described in Experiment 1 to a new group of 16 children, aged 8 to 9 years, who had been classified by pretesting as good or poor readers. These tasks were administered both with the stimulus set that grouped and with the stimulus set that did not group. All procedures were identical to those described in Experiment 1, with the following exception. Half of the subjects in each group (that is, half of the good readers and half of the poor readers) were not given instruction on strategy. They were left to develop a strategy on their own. The other half of each group was given the optimal strategy for performing the task. That is, they were told to sort on the basis of the right element and to ignore the left.

\section{Results and Discussion}

Figures 7 and 8 show the results obtained with good readers and with poor readers, respectively, for the two stimulus sets in both the strategy and no-strategy conditions. A three-way ANOVA (strategy condition $\times$ reading ability $\times$ sorting task) showed a significant main effect of reading ability $[F(1,12)=6.88, p<.05]$ and sorting task $[F(3,36)=62.81, p<.001]$, and significant strategy $\times$ sorting task and reading ability $\times$ sorting task interactions $[F(3,36)=4.79, p<.01$ and $F(3.36)=$ $3.16, \mathrm{p}<.05$, respectively]. Consider first the data obtained with good readers. The left panel of Figure 7 shows sorting times for control and orthogonal tasks in the grouping and no-grouping conditions when no strategy was given. Good readers showed significantly longer sorting times in the orthogonal task than in the control task (Scheffé multiple comparison tests, $\mathrm{p}<.05$ ) in the

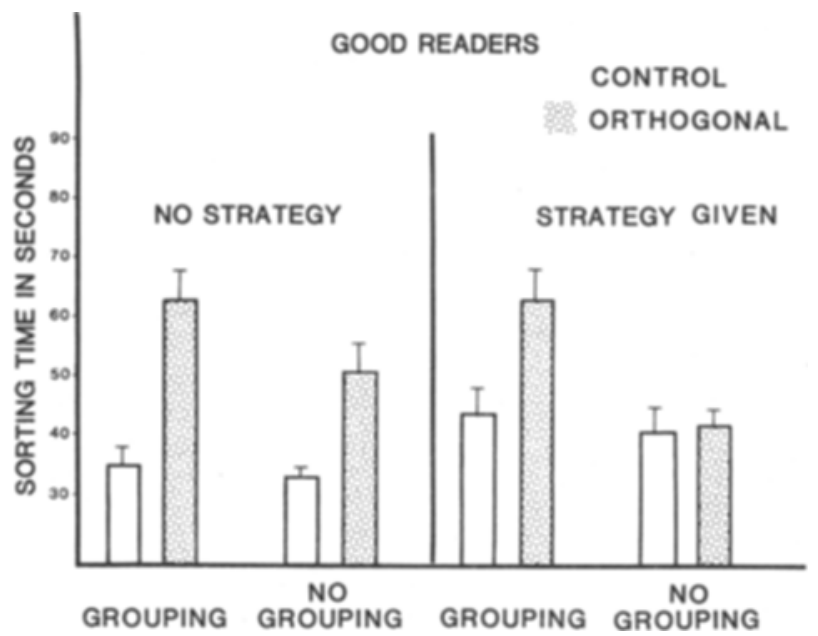

Figure 7. Data obtained with good readers in Experiment 2. Sorting times (in sec) for control and orthogonal conditions with the stimulus set that grouped and with the one that did not, shown for the no-strategy condition (left panel) and for the strategy-given condition (right panel).

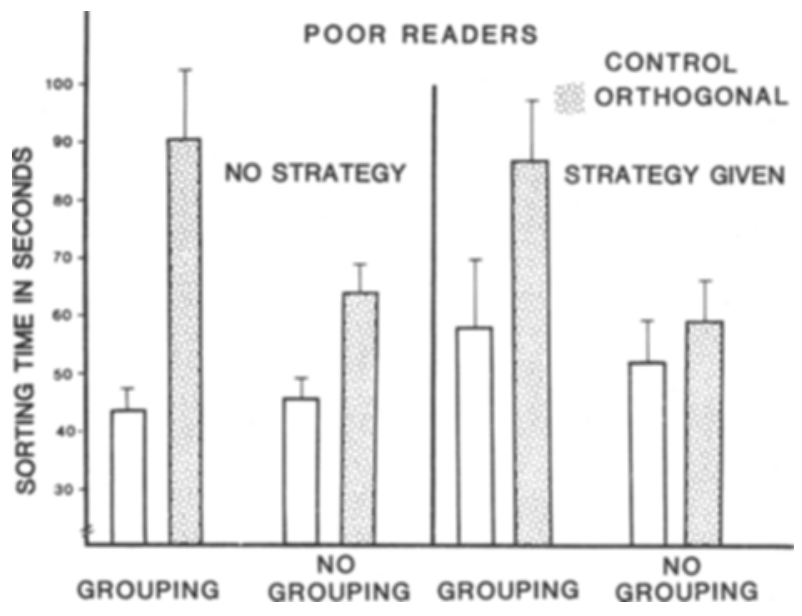

Figure 8. Data obtained with poor readers in Experiment 2. Sorting times (in sec) for control and orthogonal conditions with the stimulus set that grouped and with the one that did not, shown for the no-strategy condition (left panel) and for the strategy-given condition (right panel).

grouping condition. This increase in sorting time in the orthogonal condition is in agreement with adult data, and would ordinarily be taken as an index of the perceptual grouping effect. There was also, however, a significant increase in orthogonal over control sorting times in the no-grouping condition (Scheffé multiple comparison tests, $\mathrm{p}<.05$ ). This finding is not in agreement with adult data; although adults showed considerable ability to selectively attend to the relevant element in the orthogonal task, children did not. An increase in orthogonal over control sorting times in the no-grouping condition makes such an increase in the grouping condition a questionable measure of perceptual grouping effects.

The right panel of Figure 7 shows sorting times in the grouping and no-grouping conditions when a strategy was provided. The trends in these data resemble those obtained with adults. There is a significant increase in sorting time for the orthogonal task only with stimuli that group (Scheffé multiple comparison tests, $\mathrm{p}<.05$ ). This difference in orthogonal and control conditions can be taken as an index of perceptual grouping. It seems that children do not spontaneously develop an optimal processing strategy for selective attention, as adults do, but they are capable of selective attention if a strategy is provided.

Next, consider the data obtained with poor readers, shown in Figure 8. As with good readers, there was a significant increase in sorting times in orthogonal over control tasks, both with stimuli that group and with those that did not, when no strategy was given (Scheffé multiple comparison tests, $\mathrm{p}<.05$ ).

When a strategy was provided (right panel, Figure 8), there was a significant increase in orthogonal sorting times only in the grouping condition. Again, this difference in orthogonal and control sorting times can be taken as an index of the perceptual grouping effect. 
The above data suggest that differences in the development of a strategy for selective attention account for the different trends shown by children and adults in the nogrouping condition. When strategy is controlled, children and adults show similar trends. With this control, it becomes possible to consider the perceptual grouping effect and its relation to reading ability.

Figure 9 shows the grouping effect (the increase in orthogonal over control sorting times obtained with the stimulus set that grouped) in the strategy and no-strategy conditions. The grouping effect was stronger for poor readers, whether or not a strategy was given. A two-way ANOVA (strategy condition $\times$ reading ability), using the magnitude of the grouping effect for each subject as the dependent measure, showed a significant main effect of strategy condition $[F(1,12)=4.49, p<.05]$ and reading ability $[F(1,12)=5.05, p<.05]$. The interaction of strategy condition and reading ability was not significant, although this relationship should be investigated with a larger number of subjects per group. These results indicate that poor readers are less proficient at selective attention to elements within perceptual units, even when an optimal processing strategy is provided.

\section{GENERAL DISCUSSION}

The main finding of the present study is that poor readers show stronger perceptual grouping effects than good readers. In fact, the magnitude of perceptual grouping increases as reading ability decreases. Such an effect has implication for visual processing differences between good and poor readers. First of all, the failure of selective attention implies that a stimulus is processed as a whole or as a unit. Various theories of holistic visual processing have recently been developed. For example, it has been suggested that visual processing involves two different perceptual systems: a holistic system that



Figure 9. The grouping effect (orthogonal - control sorting times) for good and poor readers in the no-strategy condition (left panel) and in the strategy-given condition (right panel). processes large amounts of global information at high speed and relies heavily on peripheral vision, and a slower, analytic system that processes information about specific details in the visual stimulus and is accomplished mainly by foveal vision (Breitmeyer \& Ganz, 1976; Broadbent, 1977; Neisser, 1967; Weisstein, Ozog, \& Szoc, 1975). The holistic system operates preattentively (Neisser, 1967), that is, without conscious or strategic control. The analytic system is a focal attentive system, and does come under conscious control. The holistic system functions as an early warning system: it performs a crude analysis of the incoming stimulus, parses the field into regions and units, and directs the analytic system to particularly salient areas where it might be most efficacious to focus attention.

Under such a scheme, perceptual grouping would be - accomplished by the holistic, preattentive perceptual system. The fact that poor readers are arrested at the preattentive stage would then suggest that they may be more restricted to the holistic (rather than analytic) processing level. This notion finds further support in the fact that the overall magnitude of the grouping effect is diminished when an analytic processing strategy is provided. Even then, however, grouping effects are stronger for poor readers, suggesting that poor readers may be less able to exert active, strategic control of their reading behavior.

Given these findings, one would expect that poor readers would process words holistically, perhaps at the expense of the processing of detail information. The perception of detail information in words has recently been linked to the use of holistic versus analytic processing strategies (Williams, Gaffney, \& Solman, 1985). Using a word superiority/inferiority paradigm, in which holistic or analytic processing strategies can be induced, Williams et al. found that the perception of detail information in words was facilitated when processing was analytic. It may be that poor readers simply employ an inappropriate processing strategy. For example, they may process words in a holistic fashion, "holding on" to the overall percept, under conditions in which a more analytic type of processing may be necessary.

Thus, reading errors made by poor readers would more likely be based on holistic percepts of words. There is evidence, in fact, that this may be the case. From the reading data obtained in the DRS pretesting of the subjects who participated in this study, it was found that good and poor readers make distinctly different types of substitution errors. Good readers are inclined to substitute semantically similar and syntactically correct words. Poor readers, on the other hand, are more likely to decode the initial letter or letter blend, and substitute a word of similar form but irrelevant meaning. Again, it may be that these subjects lock onto an early holistic percept of a word, at the expense of the processing of detail information.

In terms of reading behavior, the above data would suggest that poor readers rely heavily on the holistic information they acquire while reading, arriving at quick decisions about the identity of words before a more detailed 
analysis is accomplished by the focal attentive mechanism. Given that the focal attentive mechanism is a process that comes under conscious or strategic control, it may be that consistent training in the employment of processing strategies could significantly influence the reading behavior of poor readers.

\section{REFERENCES}

BREITMEYER, B. , \& GANZ, L. (1976). Implications of sustained and transient channels for theories of visual pattern masking, saccadic suppression, and information processing. Psychological Review, 83, 1-36.

BRoAdBent, D. (1977). The hidden preattention process. American Psychologist, 32, 109-118.

Clifton-Everest, I. (1976). Dyslexia: Is there a disorder of visual perception? Neuropsychologia, 14, 491-494.

Critchely, M. (1964). Developmental dyslexia. London: Heinemann.

ENNs, J., \& GIRGUS, J. (1985). Perceptual grouping and spatial distortion: A developmental study. Developmental Psychology, 21, 241-246.

Lovegrove, W., \& Brown, C. (1978). Development of information processing in normal and disabled readers. Perceptual \& Motor Skills, 46, 1047-1054.

Lovegrove, W., Martin, F., \& Sl.aghuis, W. (1985). A theoretical and experimental case for a visual deficit in specific reading disability. Manuscript submitted for publication.

Natchez, G., \& Roswell, F. (1964). Reading disability: Diagnosis and treatment. New York: Basic Books,

Neisser, U. (1967), Cognitive psychology. New York: Appleton Century-Crofts.

Pick, A., \& Frankel, G. (1973). A study of strategies of visual attention in children. Developmental Psychology, 9, 348-358.

Pomerantz, J. (1977). Perceptual organization in information processing. In M. Kubovy \& J. Pomerantz (Eds.), Perceptual organization. Hillsdale, NJ: Erlbaum.

Pomerantz, J., \& Garner, W. (1973). Stimulus configuration in selective attention tasks. Perception \& Psychophysics, 14, 565-569.

Pomerantz, J., \& SchWaitzberG, S. (1975). Grouping by proximity: Selective attention measures. Perception \& Psychophysics, 18, 355-361.

SPAChE, G. (1981). Diagnostic reading scales. Monterrey, CA: McGraw-Hill.

Stanley, G. (1975). Visual memory processes in dyslexia. In D. Deutsch \& J. Deutsch (Eds.), Short-term memory. New York: Academic Press.

Stanley, G., \& Hall, R. (1973a). A comparison of dyslexics and normals in recalling letter arrays after brief presentations. British Journal of Educational Psychology, 43, 301-304.

Stanley, G., \& Hall, R. (1973b). Short-term visual information processing in dyslexia. Child Development, 44, 841-844.

Strutt, G., ANDERSON, D., \& Well, A. (1975). A developmental study of the effects of irrelevant information on speeded classification. Journal of Experimental Child Psychology, 20, 127-135.

Vellutino, F. (1977). Alternative conceptualizations of dyslexia: Evidence in support of verbal-deficit hypothesis. Harvard Educational Review, 47, 334-354.

Vellutino, F., Pruzek, R., Steger, J., \& Meshoulam, U. (1973). Immediate visual recall in poor and normal readers as a function of orthographic-linguistic familiarity. Cortex, 9, 370-386.

Vellutino, F., Steger, J., DeSetto, L., \& Phillips, F. (1975). Immediate and delayed recognition of visual stimuli in poor and normal readers. Journal of Experimental Child Psychology. 19, 223-232.

Vellutino, F., Steger, J., Kaman, M., \& DeSetto, L. (1975). Visual form perception in deficient and normal readers as a function of age and orthographic-linguistic familiarity. Cortex, 11, 22-30.

VurPILLOT, E. (1976). The visual world of the child. New York: International Universities Press.

Weisstein, N., Ozog, G., \& Szoc, R. (1975). A comparison and elaboration of two models of metacontrast. Psychological Review, 82, 325-342.

Williams, M., Gaffney, J., \& Solman, R. (1985). The word superiority effect under conditions that approximate reading. Brain \& Language, 25, 160-166.

Williams, M., \& Weisstein, N. (1980). Perceptual grouping produces spatial-frequency specific effects on metacontrast. Investigative Ophthalmology \& Visual Science, Supplement, 165.

\section{NOTES}

1. The DRS gives measures of both oral and silent reading ability. It is commonly assumed that oral and silent reading are different processes, but there is general disagreement concerning which is a better index of reading ability. We chose to classify our subjects according to silent reading ability. It should be noted, however, that our data showed the same trends when subjects were organized according to oral reading ability: the perceptual grouping effect increased as reading ability decreased.

2. For conditions involving bracket stimuli, the order of exposure of stimulus elements varies according to whether the deck is held in the left or the right hand. For example, in conditions in which the left element is irrelevant and the right element is relevant, the irrelevant element becomes visible first when the deck is held in the left hand, and the relevant element becomes visible first when the deck is held in the right hand. It has previously been shown that order of exposure does not significantly affect sorting items in selective-attention tasks (Pomerantz \& Schwaitzberg. 1975). We therefore allowed subjects to hold the deck in whichever hand they preferred. For the color sorting task, preferred hand was not a factor.

(Manuscript received April 29, 1985; revision accepted for publication September 23, 1985.) 